\title{
GENERATION OF FEW-CYCLE LASER PULSES USING A PHOTONIC QUASI-CRYSTAL FIBER
}

\footnotetext{
${ }^{1,2}$ M. S. Aruna Gandhi, ${ }^{1}$ Melwin G, ${ }^{1}$ P. Ramesh Babu, ${ }^{3}$ Abdosllam M. Abobaker, ${ }^{4}$ K. Nakkeeran and ${ }^{* 1}$ K. Senthilnathan

${ }^{1}$ School of Advanced Sciences, VIT University, Vellore-632 014, Tamil Nadu, India.

${ }^{2}$ School of Engineering, Presidency University, Bengaluru-560 089, Karnataka, India.

${ }^{3}$ Department of Communications Engineering, College of Electronic Technology, Bani Walid, Libya.

${ }^{4}$ School of Engineering, Fraser Noble Building, University of Aberdeen, Aberdeen, AB24 3UE, UK.

*senthee@gmail.com.
}

\begin{abstract}
We explore the optical properties of a proposed solid-core photonic quasi-crystal fiber (SC-PQF) of 10fold for the wavelengths from 300 to $1100 \mathrm{~nm}$. The proposed SC-PQF exhibits a admissible low dispersion of $-8.6481 \mathrm{ps}^{2} / \mathrm{km}$, a third order dispersion of $0.00415 \mathrm{ps}^{3} / \mathrm{km}$ and a large nonlinearity of $1684 \mathrm{~W}^{-1} \mathrm{~km}^{-1}$ at $450 \mathrm{~nm}$, which turn out to be the desired optical properties for generating the few-cycle laser pulses. By exploiting these optical properties, we numerically demonstrate the generation of fewcycle laser pulses at $450 \mathrm{~nm}$ wavelength by means of higher-order soliton effect compression.
\end{abstract}

\section{KEY WORDS}

Few-cycle pulse, photonic quasi-crystal fiber, Dispersion, Nonlinearity.

\section{Introduction}

In recent years, there has been a considerable interest for generating laser pulses comprising a less number of field oscillations called few-cycle laser pulses at different wavelengths. Few-cycle pulses in the femtosecond range are highly useful in the development of femtosecond laser technology. It should be emphasized that these few-cycle pulses help probe the dynamics of electrons in the matter. It is anticipated that the attosecond laser technology shall led to attoelectronics [1]. Thus, the high-intensity few-cycle laser pulses are at the forefront of the recent research in the ultrafast laser physics. 
In general, fiber-based pulse compression techniques are smartly used for generating the few-cycle laser pulses. In recent years, the generation of few and single cycle pulses in highly nonlinear photonic crystal fibers (PCFs) has been numerically studied using soliton effect pulse compression [2-4]. More recently, the higher order soliton pulse compression technique has been deployed in a waveguide [5-7]. It is demonstrated that the optical properties of PCFs could be engineered by varying the structural parameters such as pitch and diameter of the air-holes. Nonetheless, it is highly difficult to study the optical properties in PCFs for a wide range of wavelengths [8]. The recent invention of a special fiber known as photonic quasi-crystal fiber (PQF) which has the ability to surpass the limitations of the PCFs. For the first time, Kim et al. have proposed the idea of $\mathrm{PQF}$ which exhibits the unique optical properties [9]. It is reported that PQF provides interesting optical characteristics such as a scalable dispersion, a low confinement loss, a large nonlinearity and a maximum cut off ratio that can help sustain single mode condition [10].

\section{Geometric Design of SC-PQF}

Fig. 1 depicts the 2D geometrical structure of the 10 -fold SC-PQF. The perfectly matched layer is designed for calculating the confinement loss. The 10-fold SC-PQF design has the basis of penrose quasi-crystal structure [11]. Here, the diameter of the air hole, d, is chosen as $0.9 \mu \mathrm{m}$ and pitch, $\Lambda$, the distance between the two air holes as $1.5 \mu \mathrm{m}$. The perfectly matched layer is designed for calculating the confinement loss. We calculate the effective refractive index $\left(n_{\text {eff }}\right)$ for computing the waveguiding properties for the wavelengths from 300 to $1100 \mathrm{~nm}$.

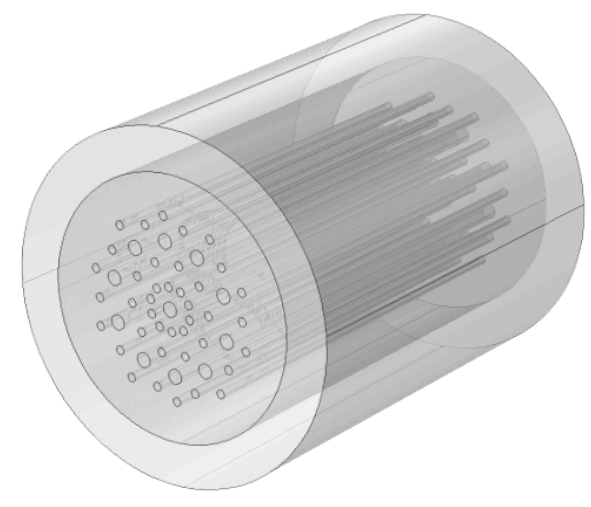

Fig. 1 Geometrical structure of the proposed SC-PQF. 


\section{Optical Properties of SC-PQF}

Here, we aim at achieving desired optical properties, namely, single mode condition, confinement loss, second order dispersion, third order dispersion and nonlinearity. Hence, we first calculate the effective refractive index $\left(n_{\text {eff }}\right)$ for the proposed SC-PQF.

\subsection{Dispersion}

Having computed the effective index, we next compute the second derivative of the real part of effective refractive index of the fundamental mode for calculating dispersion. Fig. 2 illustrates the variation of group velocity dispersion (GVD) over a range of wavelengths from 300 to $1100 \mathrm{~nm}$. We find that the proposed fiber exhibits both anomalous and normal GVD. In Fig.2, one can observe anomalous GVD from 300 to $930 \mathrm{~nm}$ and normal GVD from 940 to $1100 \mathrm{~nm}$. We have been able to achieve a less GVD of $-8.6481 \mathrm{ps}^{2} / \mathrm{km}$ at $940 \mathrm{~nm}$ wavelength for the proposed SC-PQF. This optical property turns out to be one of crucial requirements for generating few-cycle pulses.

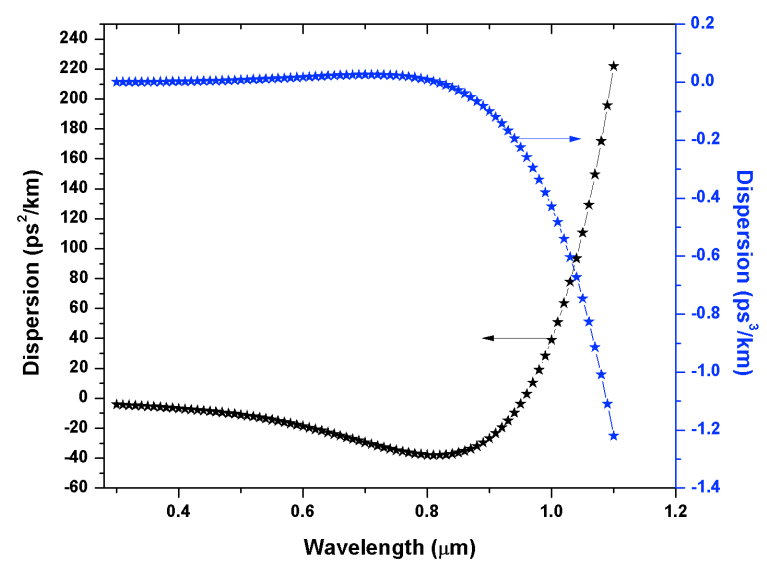

Fig. 2 GVD and TOD as a function of wavelength.

\subsection{Effective Area and Nonlinearity}

In this section, we intend computing the nonlinearity by calculating effective area. Hence, we compute the effective mode field area by calculating the effective refractive index of fundamental mode. We obtain a large nonlinearity of $1684 \mathrm{~W}^{-1} \mathrm{~km}^{-1}$ with effective mode field area of $2.65226 \mu \mathrm{m}^{2}$ at $450 \mathrm{~nm}$ wavelength. Fig. 3 illustrates the variation of nonlinearity with respect to wavelength. It is observed that 
the effective area of the fundamental mode increases as and when the wavelength is increased. On the other hand, the nonlinearity shows the opposite trend with wavelength. It is because the mode confinement becomes tight at lower wavelengths and vice-versa.

\section{Pulse Compression using SC-PQF}

By exploiting the above discussed optical properties, in this section, we delineate the generation of fewcycle pulses at various wavelengths using higher-order soliton pulse compression technique. It is well demonstrated that the fundamental optical soliton is a light pulse which is generated when the chirp produced by the anomalous dispersion gets dynamically balanced by the chirp introduced by the selfphase modulation. The temporal shape of this fundamental soliton does not change during the course of propagation. However, the temporal shape of the higher-order soliton does change, i.e., it undergoes periodic compression and broadening of its temporal width during the propagation [12]. Thus, the compression can easily be achieved by finding an optimum length of the fiber.

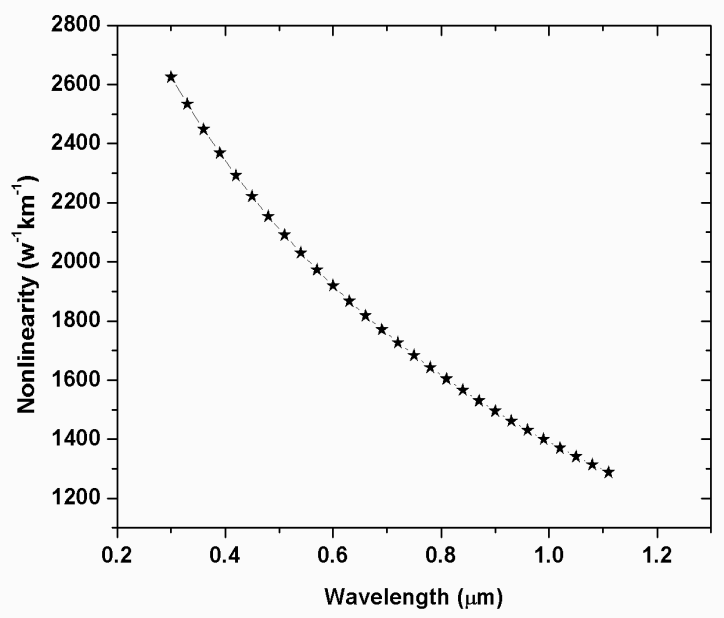

Fig. 3 Nonlinearity as a function of wavelength.

Here, the split-step Fourier method is employed for solving the HNLSE numerically. The HNLSE is given by [13],

$$
\frac{\partial A}{\partial z}+\frac{i \beta_{2}}{2} \frac{\partial^{2} A}{\partial T^{2}}-\frac{i \beta_{3}}{6} \frac{\partial^{3} A}{\partial T^{3}}=i \gamma\left[|A|^{2} A+\frac{i}{\omega_{0}} \frac{\partial}{\partial T}\left(\left|A^{2}\right| A\right)-T_{R} A \frac{\partial|A|^{2}}{\partial T}\right],
$$


where $A$ is the slowly varying amplitude of the pulse envelope, $z$ is the distance, $T$ is the time, $\beta_{2}$ and $\beta_{3}$ are second-order and third-order dispersions, respectively, $\gamma$ is the nonlinear coefficient and $T_{R}$ is the response time due to Raman effect. We consider the following chirp-free hyperbolic secant pulse as an input,

$$
A(0, T)=\sqrt{P_{0}} \operatorname{sech}\left(\frac{T}{T_{0}}\right)
$$

where $P_{0}$ and $T_{0}$ are the peak power and the input soliton duration, respectively. An hyperbolic secant pulse $A(0, T)$ is propagated into the fiber with $\beta_{2}=-8.6481 \mathrm{ps}^{2} / \mathrm{km}, \gamma=1684 \mathrm{~W}^{-1} \mathrm{~km}^{-1}$. The width of input pulse is 15 fs. Fig. 4 (a) \& (b) depicts the compression of an input pulse of width $15 \mathrm{fs}$ at $450 \mathrm{~nm}$ wavelength using a SC-PQF. The joint action of retarded optical nonlinearity and dispersion can also suppress oscillations on the temporal envelope of high-intensity femtosecond light pulses in the proposed SC-PQF.

Fig. 4 (a) explains the number of oscillations of the input pulse of 5.34 cycles. Fig. 4 (b) represents the output pulse which consists of 1.4 cycles with less than $4 \mathrm{fs}$ at $450 \mathrm{~nm}$.

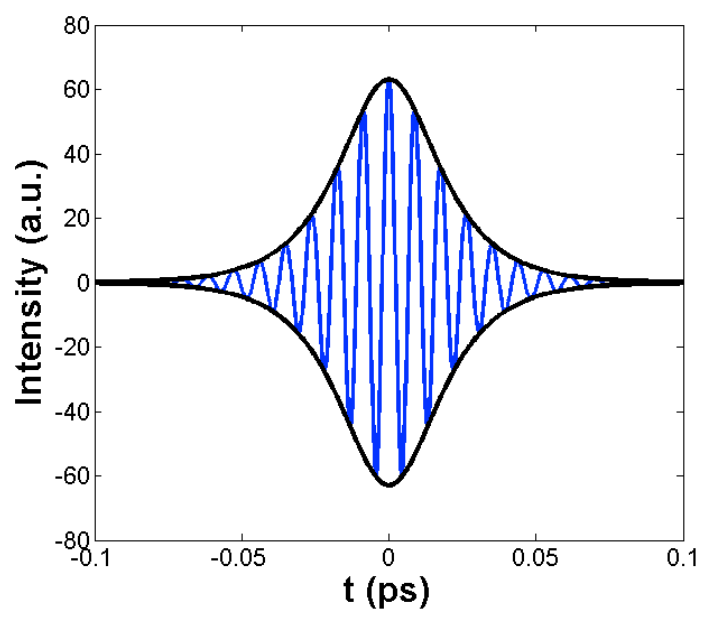

(a)

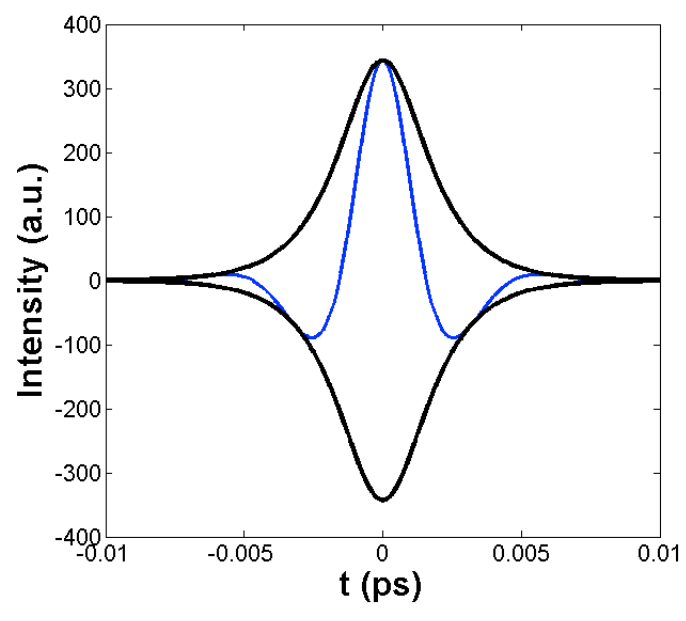

(b)

Fig.4 (a) Input and (b) Output pulses encompassing field oscillations at $450 \mathrm{~nm}$ wavelength.

\section{Conclusion}

The waveguiding properties of a SC-PQF with a solid-core have been investigated. It has been found that the proposed SC-PQF exhibits the desired optical properties over a wide range of wavelengths from 
300 to $1100 \mathrm{~nm}$. Further, we have achieved a low dispersion $\left(\beta_{2}=-8.6481 \mathrm{ps}^{2} / \mathrm{m}\right)$ and an enhanced nonlinearity $\left(\gamma=1684 \mathrm{~W}^{-1} \mathrm{~m}^{-1}\right)$ at $450 \mathrm{~nm}$ wavelength. The soliton effect compression of the femtosecond pulses in the proposed SC-PQF at $450 \mathrm{~nm}$ wavelength has also been numerically studied. The crux of the work lies in a 15 fs pulse having been compressed to 4 fs encompassing 1.4 cycles of pulse generation. We are of the opinion that the reported fiber could be used for the generation of supercontinuum.

\section{Acknowledgements}

KSN wishes to thank CSIR [No: 03(1264)/12/EMR-11] for the financial support through the project.

\section{References}

[1] F. X. Kärtner, Few-cycle laser pulse generation and its applications. (Springer Science \& Business Media, 2004).

[2] M. Foster, A. Gaeta, Q. Cao, \& R. Trebino, Soliton-effect compression of supercontinuum to fewcycle durations in photonic nanowires, Optics Express 13(18), 2005, 6848-6855.

[3] A. A. Voronin \& A. M. Zheltikov, Solitons evolving toward few- and single-cycle pulses in photonic-crystal fibers, Laser Physics 18(12), 2008, 1416-1419.

[4] A. A. Amorim, M. V. Tognetti, P. Oliveira, J. L. Silva, L. M. Bernardo, F. X. Kärtner, \& H. M. Crespo, Sub-two-cycle pulses by soliton self-compression in highly nonlinear photonic crystal fibers, Optics Letters 34(24), 2009, 3851-3853.

[5] K. Chan \& W. Cao, Improved soliton-effect pulse compression by combined action of negative third-order dispersion and Raman self-scattering in optical fibers, Journal of Optics Society of America $B, 15(9), 1998,2371-2375$.

[6] M. Y. Chen, H. Subbaraman, \& Ray T. Chen, One stage pulse compression at $1554 \mathrm{~nm}$ through highly anomalous dispersive photonic crystal fiber, Optics Express, 19(22), 2011, 21809-21817.

[7] Qian Li, J. Nathan Kutz, \& P. K. A. Wai, Cascaded higher-order soliton for non-adiabatic pulse compression, Journal of Optics Society of America B, 27(11), 2010, 2180-2189.

[8] Cerqueira Jr, S. Arismar, Recent progress \& novel applications of photonic crystal fibers, Reports on Progress in Physics, 73(2), 2010, 024401.

[9] Kim, Soan, Chul-Sik Kee, \& Jongmin Lee, Novel optical properties of six-fold symmetric photonic quasicrystal fibers, Optics Express 15(20), 2007, 13221-13226. 
[10] Han Zhao, Remo Proietti Zaccaria, Prabhat Verma, Junfeng Song, \& HongBo Sun, Validity of the V parameter for photonic quasi-crystal fibers, Optics Letters 35(7), 2010, 1064-1066.

[11] Barak Freedman, Guy Bartal, Mordechai Segev, Ron Lifshitz, Demetrios N. Christodoulides \& Jason W. Fleischer, Wave and defect dynamics in nonlinear photonic quasicrystals. Nature 440(7088), 2006, 1166-1169.

[12] R. Vasantha Jayakantha Raja, K. Senthilnathan, K. Porsezian \& K. Nakkeeran, Efficient pulse compression using tapered photonic crystal fiber at $850 \mathrm{~nm}$, IEEE J. Quantum Electron., 46(12), 2010, 1795-1803.

[13] Agrawal, Govind P. Nonlinear fiber optics (Academic press, 2013). 